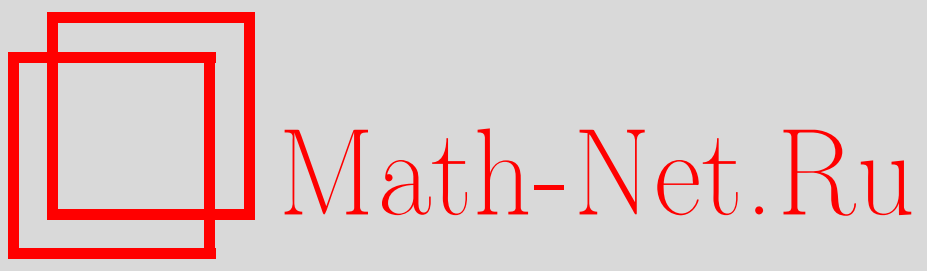

В. Д. Седых, Некоторые инварианты допустимых гомотопий пространственных кривых, Функи. анализ и его прил., 2001, том 35, выпуск 4, 54-66

DOI: https://doi.org/10.4213/faa273

Использование Общероссийского математического портала MathNet.Ru подразумевает, что вы прочитали и согласны с пользовательским соглашением

http://www . mathnet.ru/rus/agreement

Параметры загрузки:

IP : 54.210 .77 .194

26 апреля 2023 г., 15:03:37

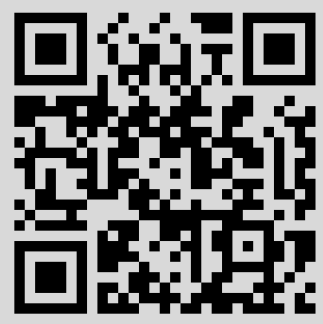




\title{
Некоторые инварианты допустимых гомотопий пространственных кривых
}

\author{
(C) 2001. В. Д. СЕдыХ
}

\section{§1. Введение}

Для каждой точки $C^{\infty}$-гладкой кривой в трехмерном вещественном проективном пространстве $P^{3}$ существует плоскость, пересекающая кривую в этой точке по меньшей мере три раза. Такая плоскость называется соприкасающейся плоскостью к кривой в данной точке. Точка кривой является ее точкой уплощения, если кратность пересечения кривой с соприкасающейся плоскостью в этой точке больше трех. Точка уплощения кривой, в которой соприкасающаяся плоскость не единственна, называется точкой перегиба.

Предположим, что для любых двух точек (взятых с учетом кратностей) гладкой замкнутой кривой в $P^{3}$ существует плоскость, которая проходит через эти точки и нигде более не пересекает кривую. Тогда эта кривая имеет не менее четырех геометрически различных точек уплощения [3].

Заметим, что кривая, удовлетворяющая указанному условию, не имеет точек перегиба, вложена и аффинна. Более того, такая кривая лежит на границе своей аффинной выпуклой оболочки. Оказывается (см. [6]), что любая гладкая замкнутая кривая, вложенная в аффинное трехмерное пространство $\mathbb{R}^{3}$ без точек перегиба и лежащая на границе своей выпуклой оболочки, имеет не менее четырех геометрически различных точек уплощения.

Было бы интересно распространить теорему о четырех точках уплощения на более широкий класс пространственных кривых. Один из подходов к решению этой задачи предложен В. И. Арнольдом в [1]. Этот подход основан на методах контактной геометрии.

Рассмотрим замкнутый фронт общего положения в $P^{3}$. Это особая поверхность с ребрами возврата, ласточкиными хвостами и трансверсальными самопересечениями. Объединение ребер возврата и вершин ласточкиных хвостов называется линией возврата фронта. Эта кривая не имеет самопересечений, но имеет точки возврата в вершинах ласточкиных хвостов.

Замыкание множества точек самопересечения фронта является объединением кривых, которые могут иметь лишь точки возврата, общие двойные или тройные самопересечения и концевые точки в вершинах ласточкиных хвостов. Эти кривые называются двойными линиями фронта. Вершины ласточкиных хвостов образуют связанную napy, если они являются концевыми точками некоторой двойной линии фронта.

Рассмотрим гладкую замкнутую кривую общего положения в $P^{3}$. Множество касательных плоскостей к кривой представляет собой замкнутый фронт общего

* Работа частично поддержана грантами РФФИ 99-01-01109, EPSRC GR K99015 и NWO-RFBR 047-008-005. 
положения в двойственном пространстве. Линия возврата этого фронта является двойственной кривой (множеством соприкасающихся плоскостей к исходной кривой). Точки уплощения кривой взаимно однозначно соответствуют вершинам ласточкиных хвостов фронта (т.е. точкам возврата двойственной кривой).

ОПРЕДЕЛЕНИЕ 1.1. Две точки уплощения гладкой замкнутой кривой общего положения в $P^{3}$ образуют связанную пару, если соответствующие вершины ласточкиных хвостов фронта касательных плоскостей к этой кривой образуют связанную пару.

Ориентируем данную кривую и объемлющее пространство. Росток кривой в точке уплощения определяется формулами

$$
x=t, \quad y=t^{2}+\ldots, \quad z=t^{4}+\ldots
$$

в подходящей системе аффинных координат. Точка уплощения называется положительной (отрицательной), если эта система координат ориентирует объемлющее пространство положительно (отрицательно).

Число связанных пар точек уплощения, имеющих один и тот же знак, не зависит от выбора ориентаций. Это число называется штурмовостью кривой. Согласно [1], штурмовость является инвариантом так называемых допустимых гомотопий кривой.

ОПРЕДЕЛЕНИЕ 1.2. Регулярная гомотопия гладкой замкнутой кривой общего положения в $P^{3}$ называется допустимой, если она определяет общее однопараметрическое семейство кривых, в котором каждая кривая не имеет

1) самопересечений (т. е. ее фронт не имеет самокасаний);

2) точек перегиба (т. е. двойственная кривая неприводима);

3) касаний с гладкой частью развертки своих касательных (т. е. ребра возврата фронта не касаются его гладкой части);

4) касательных плоскостей, соприкасающихся с кривой в двух различных точках (т. е. двойственная кривая не имеет самопересечений).

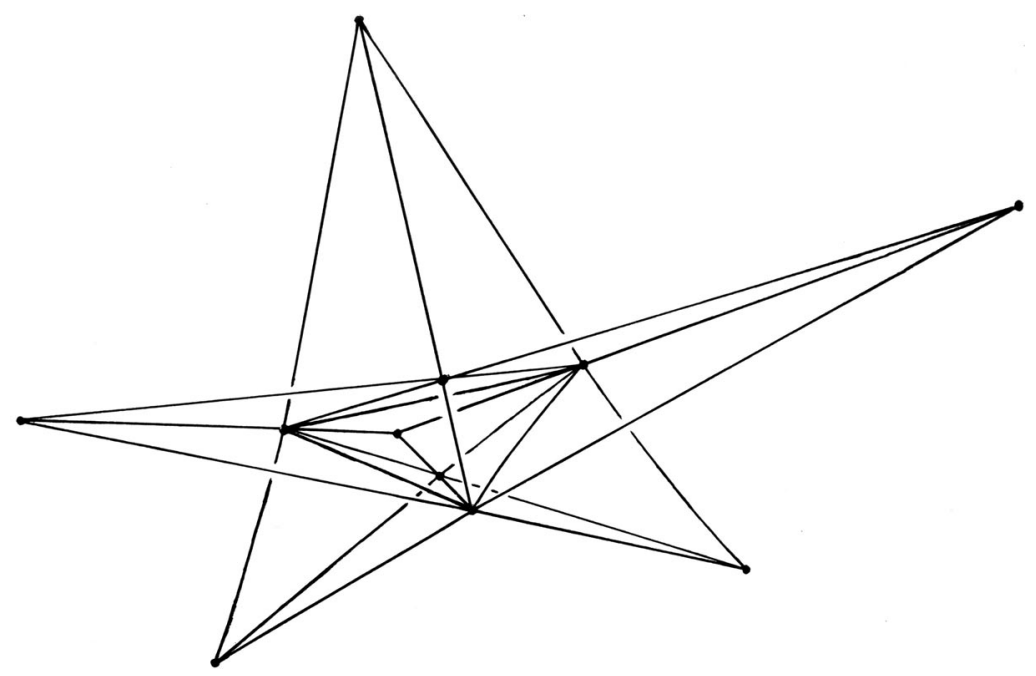

Рис. 1 
В процессе допустимых гомотопий число точек уплощения кривой не может стать меньше удвоенной штурмовости. Но штурмовость может быть равна нулю. Рассмотрим, например, кривую

$$
\Gamma: x=\cos t, y=\sin t, z=\cos 3 t, \quad t \bmod 2 \pi .
$$

Множество особых точек ее фронта (линия возврата и самопересечения) изображено на рис. 1 (детали см. в §6). Кривые общего положения, достаточно близкие к $Г$ (в $C^{\infty}$-топологии), имеют 6 точек уплощения. Некоторые из них имеют нулевую штурмовость. В. И. Арнольд поставил следующий вопрос (см. [2, задача 1998-6]): можно ли уничтожить допустимыми гомотопиями все 6 точек уплощения таких кривых?

Мы даем ниже отрицательный ответ на этот вопрос, а именно, мы показываем, что число точек уплощения любой кривой общего положения, достаточно близкой к Г, не может обратиться в нуль в процессе допустимых гомотопий. Для доказательства мы определяем новый инвариант допустимых гомотопий пространственных кривых - число замкнутых двойных линий фронта. Кроме того, мы строим инвариант, обобщающий штурмовость кривой. Это хордовая диаграмма, в которой число хорд, пересекающих нечетное число других хорд, равно штурмовости кривой.

\section{§2. Замкнутые двойные линии фронта}

Пусть $\gamma: S^{1} \rightarrow P^{3}-$ гладкая замкнутая кривая общего положения в $P^{3}$ и $l-$ двойная линия ее фронта. Ориентируем двойственное пространство $P^{3 *}$, двойственную кривую $\gamma^{*}$ и линию $l$. Точка возврата $c$ на $l$ является положительной (отрицуательной), если следующий набор трех векторов в $T_{c} P^{3 *}$ положительно (отрицательно) ориентирован:

1) касательный вектор к $\gamma^{*}$;

2) направляющий вектор односторонней полукасательной к $l$ в $c$;

3) вектор в касательной плоскости к гладкой части фронта $\gamma$ в $c$, указывающий направление отклонения ветви $l$, выходящей из $c$, от прямой, касающейся $l$ в $c$.

ОПРЕДЕЛЕНИЕ 2.1. Абсолютная величина разности между числом положительных и числом отрицательных точек возврата на линии $l$ называется весом этой линии.

Вес линии $l$ не зависит от выбора ориентаций.

ЗАмЕЧАниЕ 2.2. Если линия $l$ не является замкнутой и ее концы соответствуют точкам уплощения кривой $\gamma$ противоположных знаков, то вес линии $l$ может быть определен со знаком. Действительно, ориентации пространства $P^{3 *}$ и кривой $\gamma^{*}$ индуцируют ориентации пространства $P^{3}$ и кривой $\gamma$ и определяют естественную ориентацию линии $l$ : из конца, отвечающего отрицательной точке уплощения кривой $\gamma$, в конец, отвечающий положительной точке уплощения. Если зафиксировать такую ориентацию линии $l$, то разность между числом положительных и числом отрицательных точек возврата на $l$ не будет зависеть от выбора ориентаций пространства $P^{3 *}$ и кривой $\gamma^{*}$.

Теорема 2.3. Набор (неупорядоченный) весов замкнутых двойных линий фронта гладкой замкнутой кривой общего положения в $P^{3}$ является инвариантом допустимых гомотопий. В частности, число этих линий не меняется при таких гомотопиях. 


\section{Доказательство приведено в $§ 4$.}

ОПрЕДЕЛЕНИЕ 2.4. Две гладкие замкнутые кривые, вложенные в $P^{3}$, называются изотопнылми, если они гомотопны в пространстве вложенных кривых.

Допустимые гомотопии сохраняют изотопический класс кривой.

Теорема 2.5. Пусть гладкая замкнутая кривая общзего положения в $P^{3}$ изотопна аффинной. Тогда ее фронт имеет двойнье линии (m. е. существуют проективные плоскости в $P^{3}$, касающиеся кривой в двух различных точках).

Если кривая аффинна, то это очевидно. Действительно, такая кривая имеет аффинные опорные плоскости, касающиеся ее в двух различных точках (см. [5]). Доказательство в общем случае дано в $\$ 5$.

ЗАмЕчАниЕ 2.6. Если кривая в $P^{3}$ не изотопна аффинной, то ее фронт может не иметь двойных линий. Например, выпуклая кривая

$$
(\cos t: \sin t: \cos 3 t: \sin 3 t), \quad t \bmod \pi,
$$

пересекает любую плоскость не более, чем в трех точках (с учетом кратностей). Эта кривая нестягиваема. Кривая

$$
(\cos t: \sin t: \cos 2 t: \sin 2 t), \quad t \bmod 2 \pi,
$$

стягиваема, но также не имеет двойных касательных плоскостей. Мы покажем в $\S 5$, что она не изотопна аффинной.

Из теорем 2.3 и 2.5 имеем

СледствиЕ 2.7. Пусть гладкая замкнутая кривая общего положения в $P^{3}$ изотопна аффинной и ее фронт не имеет замкнутых двойных линий. Тогда эта кривая, как и любая другая кривая, полученная из нее допустимьми гомотопиями, имеет точки уплощения.

Рассмотрим кривую Г из $\$ 1$. Она аффинна.

ПрЕДЛОЖЕНИЕ 2.8. Фронт любой кривой общего положения, достаточно близкой к Г, не имеет замкнутых двойньх линий.

Доказательство приведено в $§ 6$. Из предложения 2.8 и следствия 2.7 получаем ответ на упомянутый выше вопрос Арнольда:

СледСТВИЕ 2.9. Число точек уплощения любой кривой общего положения, достаточно близкой $\kappa$ Г, не может обратиться в нуль в процуессе допустимьх гомотопий.

\section{§3. Диаграмма главных уплощений кривой}

Рассмотрим точки уплощения гладкой замкнутой кривой $\gamma$ общего положения B $P^{3}$.

ОПРЕДЕЛЕНИЕ 3.1. Весом связанной пары точек уплощения кривой $\gamma$ называется вес двойной линии фронта кривой, которая связывает соответствующие вершины ласточкиных хвостов.

Две связанные пары точек уплощения перемежаются, если при обходе вокруг кривой точка уплощения одной связанной пары следует за точкой уплощения другой. Точки уплощения перемежающихся связанных пар называются основнылми точками уплощения рассматриваемой кривой.

ОПРЕДЕЛЕНИЕ 3.2. Связанная пара точек уплощения кривой называется неглавной, если ее вес равен 2 и точки этой пары разбивают кривую на две открытые 
дуги, одна из которых не имеет основных точек уплощения. Все остальные связанные пары называются главными.

Кривая $\gamma$ определяет весовую хордовую диаграмму $D_{\gamma}$ неупорядоченных пар точек на $S^{1}$, являющихся прообразами главных связанных пар точек уплощения и снабженных весами этих пар. Две такие диаграммы эквивалентны, если одна из них может быть переведена в другую диффеоморфизмом $S^{1}$, сохраняющим ориентацию.

ОПРЕДЕЛЕНИЕ 3.3. Класс эквивалентности диаграммы $D_{\gamma}$ называется диаграммой главных уплощуений кривой $\gamma$.

ЗАмЕчАНИЕ 3.4. Штурмовость кривой равна числу связанных пар точек уплощения, которые перемежаются с нечетным числом других связанных пар, т.е. числу хорд диаграммы главных уплощений, которые пересекают нечетное число других хорд. В частности, штурмовость кривой не превосходит числа всех хорд диаграммы главных уплощений, т.е. не превосходит числа главных связанных пар точек уплощения.

Теорема 3.5. Диаграмма главных уплощений гладкой замкнутой кривой общего положения в $P^{3}$ является инвариантом допустимых гомотопий. $B$ частности, число главных связанных пар точек уплощения кривой не меняется при таких гомотопиях.

Доказательство приведено в $\$ 4$.

СледствиЕ 3.6. В процессе допустимых гомотопий число точек уплощуения гладкой замкнутой кривой общего положения в $P^{3}$ не может стать меньше удвоенного числа главных связанных пар точек уплощения исходной кривой.

Рассмотрим кривую $Г$ из $\$ 1$. Она находится не в общем положении (Г имеет три пары точек, в которых соприкасающиеся плоскости совпадают).

ПреДЛОЖЕНИЕ 3.7. Диаграмма главных уплощений любой кривой общцего положения $\gamma$, достаточно близкой $\kappa \Gamma$, имеет один из следующих двух типов:

1) пустая диаграмма (без хорд; кривая $\gamma$ имеет три неглавные связанные пары точек уплощения; для каждой пары двойная линия фронта, связывающуая соответствующие вериины ласточкиных хвостов, имеет две точки возврата одного и того же знака);

2) непустая диаграмма, имеющая две пересекаюшиеся хорды веса 0 (кривая $\gamma$ имеет две перемежающиеся и одну неглавную связанные пары точек уплошения; двойная линия фронта, связывающая вериины ласточкиньх хвостов, соответствующие неглавной паре, имеет две точки возврата одного и того же знака; двойная линия фронта, связьвающая вершинь ласточкиньх хвостов, соответствующие любой главной паре, имеет две точки возврата противоположных знаков).

Доказательство приведено в $\$ 6$. Из предложения 3.7 и следствия 3.6 имеем

СледствиЕ 3.8. Число точек уплощения любой кривой общего положения, достаточно близкой к Г и имеющей непустую диаграмму главных уплощений, не может стать меньше 4 в проияессе допустимьх гомотопий. 


\section{§4. Доказательство теорем 2.3 и 3.5}

Рассмотрим допустимую гомотопию гладкой замкнутой кривой общего положения в $P^{3}$. Она определяет деформацию фронта этой кривой. Перестройки фронта, которые могут происходить при такой деформации, перечислены в [1]. А именно:

$A_{4}$ : рождение или смерть связанной пары вершин ласточкиных хвостов на линии возврата фронта, которые близки друг к другу вдоль этой линии (двойная линия фронта, которая их связывает, имеет две точки возврата одного и того же знака);

$A_{3}+A_{1}$ : прохождение гладкой части фронта через вершину ласточкиного хвоста (на двойной линии фронта появляется или исчезает пара точек возврата противоположных знаков);

$A_{2}+2 A_{1}$ : прохождение ребра возврата через двойную линию фронта;

$4 A_{1}$ : прохождение гладкой части фронта через точку его тройного самопересечения;

$2 A_{1} \| A_{1}$ : касание двойной линии фронта с гладкой частью фронта.

Перестройки $A_{3}+A_{1}, A_{2}+2 A_{1}, 4 A_{1}$ и $2 A_{1} \| A_{1}$ не меняют число двойных линий фронта, их типы (замкнутая или незамкнутая линия) и веса. Перестройка $A_{4}$ изменяет число двойных линий фронта, которые связывают вершины ласточкиных хвостов, соответствующие неглавным связанным парам точек уплощения кривой. Теоремы 2.3 и 3.5 доказаны.

\section{§5. Доказательство теоремы 2.5}

Сначала мы опишем изотопический инвариант стягиваемых кривых в трехмерном проективном пространстве. Построение этого инварианта осуществляется по стандартной схеме теории узлов, изложенной в [4].

Пусть $\Omega-$ пространство гладких замкнутых стягиваемых параметризованных кривых $\gamma: S^{1} \rightarrow P^{3}$. Рассмотрим особую гиперповерхность $\Upsilon \subset \Omega$, образованную кривыми $\gamma$, для которых найдется пара точек $t_{1}, t_{2} \in S^{1}, t_{1} \neq t_{2}$, таких, что $\gamma\left(t_{1}\right)=\gamma\left(t_{2}\right)$ и каждая из частей, на которые эти точки делят окружность, отображается в замкнутую нестягиваемую кривую в $P^{3}$. Через $\Upsilon_{0}$ обозначим гладкую часть гиперповерхности $\Upsilon$.

Пусть две кривые $\gamma_{0}, \gamma_{1} \in \Omega \backslash \Upsilon$ соединены путем $\gamma_{s}, s \in[0,1]$, общего положения в пространстве $\Omega$. Этот путь может пересекать гиперповерхность $\Upsilon$ только в точках из $\Upsilon_{0}$, причем трансверсальным образом. Такие пересечения мы будем называть перестройками проективности кривой в семействе $\gamma_{s}$. Каждой перестройке проективности можно сопоставить знак как обычной перестройке двойного самопересечения кривой в трехмерном пространстве.

А именно, зафиксируем ориентацию пространства $P^{3}$ и рассмотрим кривую $\gamma_{s_{*}} \in \Upsilon_{0}$ с точкой самопересечения $\gamma_{s_{*}}\left(t_{1}\right)=\gamma_{s_{*}}\left(t_{2}\right), t_{1} \neq t_{2}$. Выберем аффинную карту $\mathbb{R}^{3} \subset P^{3}$, содержащую эту точку. Тогда для каждого $s \neq s_{*}$, достаточно близкого к $s_{*}$, определен базис $B_{s}$ в $\mathbb{R}^{3}$, образованный касательными векторами $\gamma_{s}^{\prime}\left(t_{1}\right), \gamma_{s}^{\prime}\left(t_{2}\right)$ к кривой $\gamma_{s}$ и вектором с началом $\gamma_{s}\left(t_{1}\right)$ и концом $\gamma_{s}\left(t_{2}\right)$. Перестройка кривой $\gamma_{s}$ в момент $s=s_{*}$ положительна (отрицательна), если базис $B_{s}$ положительно (отрицательно) ориентирован при $s>s_{*}$.

Коориентируем гладкую часть $\Upsilon_{0}$ гиперповерхности $\Upsilon$ ростками путей $\left(\gamma_{s}, s^{*}\right)$ с положительной перестройкой проективности при $s=s^{*}$. 
ОПрЕДЕлЕНИЕ 5.1. Алгебраическое число перестроек проективности кривой $\gamma$ общего положения при общем стягивании этой кривой в точку называется коэффициентом проективности кривой $\gamma$.

ПрЕДЛОЖЕНИЕ 5.2. Коэффициент проективности гладкой замкнутой стягиваемой кривой общего положения в $P^{3}$ не зависит от способа ее стягивания, т. е. является инвариантом кривой. В частности, коэффициент проективности не меняется при изотопиях кривой.

ДокАЗАТЕЛЬСтво. Покажем, что коэффициент проективности кривой $\gamma$ не меняется при непрерывной деформации стягивающего пути.

Действительно, определенная выше коориентация гладкой части гиперповерхности $\Upsilon$ естественным образом продолжается до коориентации локально неприводимых компонент этой гиперповерхности в точках трансверсального самопересечения. Поэтому необходимо убедиться лишь в том, что коориентации гладких частей гиперповерхности $\Upsilon$ согласованы вблизи единственного страта коразмерности 1, не сводящегося к самопресечениям, - страта, отвечающего общему самокасанию кривой. Но последнее очевидно: любая достаточно малая петля общего положения, обходящая указанный страт в его двумерной трансверсали, пересекает $\Upsilon_{0}$ ровно два раза, причем в противоположных направлениях.

Остается проверить, что алгебраическое число $I\left(\gamma_{s}\right)$ точек, в которых (произвольный) замкнутый путь $\gamma_{s}$ общего положения в пространстве $\Omega$ пересекает гладкую часть гиперповерхности $\Upsilon$, равно 0 . Для этого рассмотрим путь $\tilde{\gamma}_{s}$, состоящий из одноточечных кривых

$$
\tilde{\gamma}_{s}: S^{1} \rightarrow P^{3}, \quad \tilde{\gamma}_{s}: t \mapsto \gamma_{s}\left(t_{0}\right),
$$

определенных значениями кривых $\gamma_{s}$ в фиксированной точке $t_{0}$ окружности $S^{1}$. Так как никакая одноточечная кривая в $P^{3}$ не принадлежит гиперповерхности $\Upsilon$, то $I\left(\tilde{\gamma}_{s}\right)=0$. Следовательно, $I\left(\gamma_{s}\right)=I\left(\tilde{\gamma}_{s}\right)=0$, поскольку пути $\gamma_{s}$ и $\tilde{\gamma}_{s}$ гомотопны. Предложение 5.2 доказано.

Перейдем к доказательству теоремы 2.5. Оно основано на вычислении коэффициента проективности стягиваемой кривой без двойных касательных плоскостей (касающихся кривой в двух точках).

Пусть $\gamma$ - гладкая замкнутая кривая общего положения в $P^{3}$.

ЛЕмма 5.3. Предположим, ито фронт кривой $\gamma$ не имеет двойных линий. Тогда найдутся проективная прямая $L$ в $P^{3}$ и натуральное число $n$, такие, что любая плоскость, проходящая через $L$, пересекает $\gamma$ ровно в $n$ геометрически различных точках.

ДокАЗАТЕЛЬСтвО. Рассмотрим произвольную точку $O$ кривой $\gamma$ и касательную прямую $l$ к $\gamma$ в $O$. Кривая $\gamma$ и множество плоскостей, проходящих через прямую $l$, компактны (последнее является проективной прямой в $P^{3 *}$ ). По условию кривая $\gamma$ не имеет двойных касательных плоскостей. Следовательно, для любой окрестности $U \subset \gamma$ точки $O$ найдется окрестность $V$ прямой $l$ (в пространстве прямых в $\left.P^{3}\right)$, такая, что плоскости, проходящие через прямые из $V$, трансверсальны кривой $\gamma$ вне $U$.

Покажем, что любая окрестность прямой $l$ содержит такую прямую, что плоскости, проходящие через нее, трансверсальны кривой $\gamma$ в любой заданной окрестности $U$. Для этого выберем аффинную систему координат $(x, y, z)$ в $P^{3}$ с нача- 
лом в точке $O$, такую, что росток кривой $\gamma$ в $O$ определяется формулами

$$
x=t, \quad y=y(t)=t^{2}+\ldots, \quad z=z(t)=t^{3}+\ldots,
$$

где $t \in(-\varepsilon, \varepsilon), \varepsilon>0$.

Прямая $l_{\delta}$, заданная уравнениями $y=\delta, z=4 \delta x$, близка к прямой $l=\{y=$ $z=0\}$, если $\delta$ близко к 0 . Любая плоскость, проходящая через $l_{\delta}$, определяется уравнением $a(z-4 \delta x)+b(y-\delta)=0$ для некоторых $a, b$, таких, что $a^{2}+b^{2} \neq 0$. Эта плоскость касается кривой $\gamma$ в точке $t$, если и только если

$$
\operatorname{det}\left(\begin{array}{cc}
z(t)-4 \delta t & y(t)-\delta \\
z^{\prime}(t)-4 \delta & y^{\prime}(t)
\end{array}\right)=0 .
$$

Но указанный определитель равен $-t^{4} p(t)-\delta t^{2} q(t)-4 \delta^{2}$, где

$$
p(t)=1+\cdots>0, \quad q(t)=1+\cdots>0
$$

для всех $t \in U=(-\varepsilon, \varepsilon)$, если $\varepsilon$ достаточно мало. Следовательно, плоскости, проходящие через прямую $l_{\delta}$, трансверсальны $\gamma$ в окрестности $U$ для любого $\delta>0$. Лемма 5.3 доказана.

Если кривая $\gamma$ стягиваема (нестягиваема), то число $n$ в лемме 5.3 четно (нечетно соответственно).

ЗАмЕЧАНИЕ 5.4. Кривые в $P^{3}$, фронт которых не имеет двойных линий, обобщают понятие выпуклой кривой. Действительно, для каждой такой кривой существует натуральное число $N$, такое, что кривая пересекает любую плоскость общего положения в $N$ или $N+2$ точках. Для выпуклых кривых $N=1$.

Лемма 5.5. Пусть кривая $\gamma$ стягиваема и ее фронт не имеет двойных линий. Тогда коэффициент проективности кривой $\gamma$ не равен 0.

ДокАЗАТЕЛьствО. Рассмотрим развертку $T$ кривой $\gamma$ (объединение всех ее касательных прямых). Эта поверхность является образом гладкого отображения двумерного тора в $P^{3}$. Она не имеет самопересечений, но имеет особенности в точках кривой $\gamma$. А именно, кривая $\gamma$ является ребром возврата развертки $T$.

Пусть $L \subset P^{3}-$ проективная прямая из леммы 5.3. Тогда существует натуральное число $m$, такое, что любая плоскость, проходящая через $L$, трансверсально пересекает кривую $\gamma$ ровно в $2 m$ точках. Пересечение такой плоскости с разверткой $T$ является простой замкнутой кривой. Эта кривая аффинна (не пересекает прямую $L$ ) и имеет точки возврата в точках кривой $\gamma$.

Зафиксируем произвольную плоскость П, проходящую через прямую $L$, и обозначим через $\omega$ кривую $\Pi \cap T$. Когда точка пересечения касательной прямой $l$ к $\gamma$ с плоскостью П пробегает кривую $\omega$, точка касания прямой $l$ с кривой $\gamma$ совершает $2 m$ оборотов вокруг прямой $L$.

Следовательно, если в $P^{3}$ выбрать однородные координаты $\left(x_{0}: x_{1}: x_{2}: x_{3}\right)$ так, что $\Pi=\left\{x_{3}=0\right\}, L=\left\{x_{2}=x_{3}=0\right\}$, то развертка $T$ будет гомеоморфна тору

$$
\{(\cos \varphi: \sin \varphi: \cos \psi: \sin \psi), \varphi \bmod 2 \pi, \psi \bmod \pi\}
$$

а кривая $\gamma$ изотопна его обмотке $\alpha_{m}$, при которой каждому повороту вокруг прямой $L$ на угол $\Delta \psi=\pi$ отвечает поворот на угол $\Delta \varphi=\pi+\pi / m$ вокруг прямой $x_{0}=x_{1}=0$. Очевидно, что

$$
\alpha_{m}=\{(\cos (m+1) t: \sin (m+1) t: \cos m t: \sin m t), t \bmod 2 \pi\}
$$

с точностью до проективного преобразования, сохраняющего П и $L$. 
Пусть $A, B, C$ - любые три последовательные точки пересечения кривой $\alpha_{m}$ с плоскостью $x_{3}=0(C=A$, если $m=1)$. Тогда кривая $\alpha_{m}$ гомотопна кривой $\beta$, полученной из $\alpha_{m}$ заменой ее дуги $A B C$ дугой $A B C$ окружности $x_{0}^{2}+x_{1}^{2}=x_{2}^{2}$, $x_{3}=0$. Такая гомотопия может быть осуществлена последовательностью $2 m-1$ перестроек простого двойного самопересечения, содержащей ровно $m$ перестроек проективности одного и того же знака.

Заметим, что кривая $\beta$ изотопна кривой $\alpha_{m-1}$. Поэтому можно повторить указанную процедуру $m$ раз и получить аффинную кривую $\alpha_{0}$. Эта гомотопия будет содержать ровно $m+(m-1)+\cdots+1=m(m+1) / 2$ перестроек проективности одного и того же знака. Следовательно, коэффициент проективности кривой $\gamma$ равен $m(m+1) / 2$, т. е. отличен от 0 . Лемма 5.5 доказана.

Теорема 2.5 следует из леммы 5.5, предложения 5.2 и того факта, что коэффициент проективности аффинной кривой равен 0.

ЗАмЕЧАНИЕ 5.6. Из сказанного выше следует, что если гладкая замкнутая стягиваемая кривая общего положения в $P^{3}$ не имеет двойных касательных плоскостей, то она изотопна кривой $\alpha_{m}$ для некоторого натурального $m$. Кривые $\alpha_{m}$, $m=1,2, \ldots$, не изотопны аффинной и не имеют двойных касательных плоскостей. Кривые с различными числами $m$ не изотопны.

\section{§6. Доказательство предложений 2.8 и 3.7}

Рассмотрим кривую $\Gamma$ из $\$ 1$. Она лежит в аффинном пространстве $\mathbb{R}^{3}=$ $\{(x, y, z)\}$ и имеет строго выпуклую проекцию на плоскость $z=0$. Следовательно, любая плоскость, соприкасающаяся с Г или касающаяся ее по меньшей мере в двух различных точках, трансверсальна прямой $x=y=0$ и определяется уравнением $z=a x+b y+c$.

Возьмем коэффициенты $(a, b, c)$ в качестве аффинных координат в двойственном пространстве $\mathbb{R}^{3 *}$ и проведем некоторые вычисления.

ЛЕмма 6.1. Для любых двух точек $t_{1} u t_{2} \kappa$ кивой $\Gamma$

$$
\operatorname{det}\left(\begin{array}{lll}
x^{\prime}\left(t_{1}\right) & x^{\prime}\left(t_{2}\right) & x\left(t_{1}\right)-x\left(t_{2}\right) \\
y^{\prime}\left(t_{1}\right) & y^{\prime}\left(t_{2}\right) & y\left(t_{1}\right)-y\left(t_{2}\right) \\
z^{\prime}\left(t_{1}\right) & z^{\prime}\left(t_{2}\right) & z\left(t_{1}\right)-z\left(t_{2}\right)
\end{array}\right)=32\left(\sin \frac{t_{2}-t_{1}}{2}\right)^{4} \cos \frac{t_{2}-t_{1}}{2} \sin \frac{3}{2}\left(t_{2}+t_{1}\right)
$$

Из леммы 6.1 следует, что любые две точки $t_{1}, t_{2}$ (взятые с учетом кратностей), в которых кривая Г имеет общую касательную плоскость, удовлетворяют одному из следующих четырех уравнений $(\bmod 2 \pi)$ :

$$
t_{2}+t_{1}=\frac{2 \pi k}{3} \quad(k=0,1,2) \quad \text { и } \quad t_{2}-t_{1}=\pi .
$$

ЛЕмма 6.2. Множество плоскостей, касающихся кривой Г по меньшей мере в двух точках (взятых с учетом кратностей), является объединением четырех кривых в $\mathbb{R}^{3 *}$ :

$$
\xi_{k}:\left\{\begin{array}{l}
a=3(-1)^{k} \cos \frac{\pi k}{3}\left(4 \cos ^{2} t-1\right), \\
b=3(-1)^{k} \sin \frac{\pi k}{3}\left(4 \cos ^{2} t-1\right), \\
c=-8(-1)^{k} \cos ^{3} t
\end{array} \quad t \in[0, \pi], k=0,1,2\right.
$$




$$
\eta:\left\{\begin{array}{l}
a=2 \cos 2 t-\cos 4 t, \\
b=-2 \sin 2 t-\sin 4 t, \\
c=0
\end{array} \quad t \bmod \pi .\right.
$$

Кривые $\xi_{1}, \xi_{2}, \xi_{3}, \eta$ плоские. А именно, кривая $\xi_{k}$ лежит в вертикальной плоскости $a \sin \frac{\pi k}{3}=b \cos \frac{\pi k}{3}$, а $\eta$ лежит в горизонтальной плоскости $c=0$.

Обозначим фронт кривой $\Gamma$ через $\Sigma$.

ЛЕмма 6.3. Каждая кривая $\xi_{k}, k=0,1,2$, является частью полукубической параболь с точкой возврата

$$
C_{k}=\left(3(-1)^{k+1} \cos \frac{\pi k}{3}, 3(-1)^{k+1} \sin \frac{\pi k}{3}, 0\right) .
$$

Ее концевье точки

$$
V_{k}^{m}=\left(9(-1)^{k} \cos \frac{\pi k}{3}, 9(-1)^{k} \sin \frac{\pi k}{3}, 8(-1)^{m+1}\right), \quad m=0,1,
$$

являются вериинами ласточкиньх хвостов фронта $\Sigma$. Кривая $\eta$ является гипоцииллоидой с тремя точками возврата $C_{0}, C_{1}, C_{2}$.

Заметим, что кривые $\xi_{1}, \xi_{2}, \xi_{3}$ имеют две общие точки $T_{0}=(0,0,-1)$ и $T_{1}=(0,0,1)$. Они являются точками тройного самопересечения фронта $\Sigma$. Объединение кривых $\xi_{1}, \xi_{2}, \xi_{3}, \eta$ изображено на рис. 2 (для простоты мы изображаем точки возврата углами).

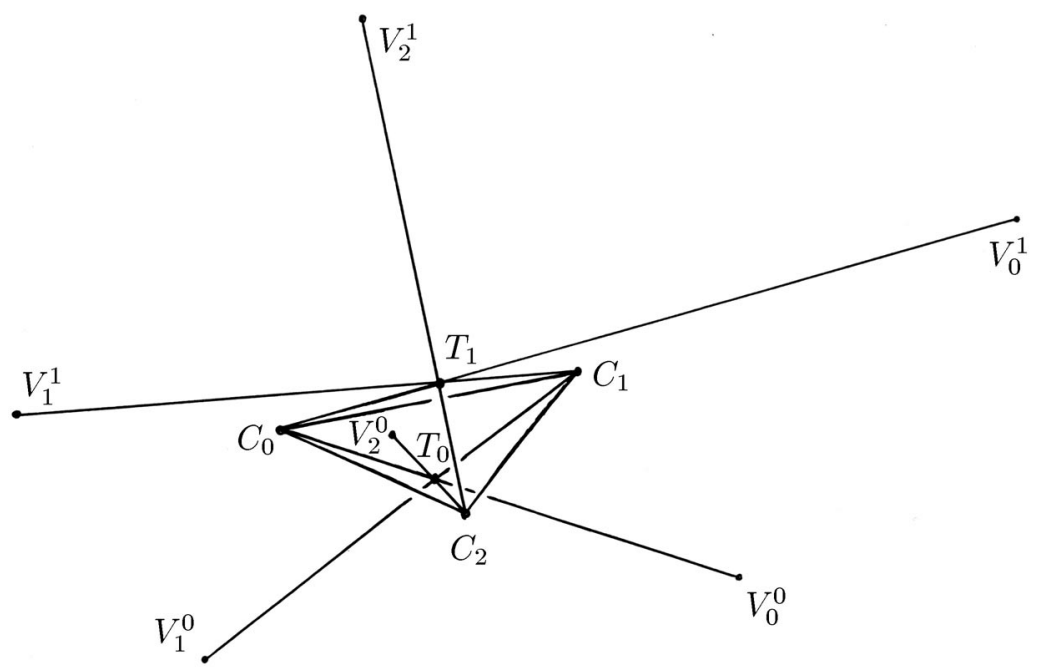

Рис. 2

Лемма 6.4. Соприкасающаяся плоскость к кривой $\Gamma$ в точке $t$ имеет следующие координаты в $\mathbb{R}^{3 *}$ :

$$
a=6 \cos 2 t+3 \cos 4 t, \quad b=3 \sin 4 t-6 \sin 2 t, \quad c=-8 \cos 3 t .
$$

Формулы леммы 6.4 определяют параметризацию двойственной кривой $\Gamma^{*}$. 
Лемма 6.5. Кривая $\Gamma^{*}$ является замкнутой кривой с шестью точками возврата $V_{k}^{m}, k \in\{0,1,2\}, m \in\{0,1\}$, и тремя точками самопересечения $C_{0}$, $C_{1}, C_{2}$. Каждая точка $C_{k}$ является точкой пересечения двух ветвей кривой $\Gamma^{*}$, причем каждая ветвь трансверсально пересекает в этой точке плоскость $c=0$ и соприкасающуюся плоскость к другой ветви.

Двойственная кривая $\Gamma^{*}$ изображена на рис. 3. Мы будем предполагать, что она ориентирована порядком своих точек возврата:

$$
V_{0}^{0} \rightarrow V_{1}^{1} \rightarrow V_{2}^{0} \rightarrow V_{0}^{1} \rightarrow V_{1}^{0} \rightarrow V_{2}^{1} \rightarrow V_{0}^{0} .
$$

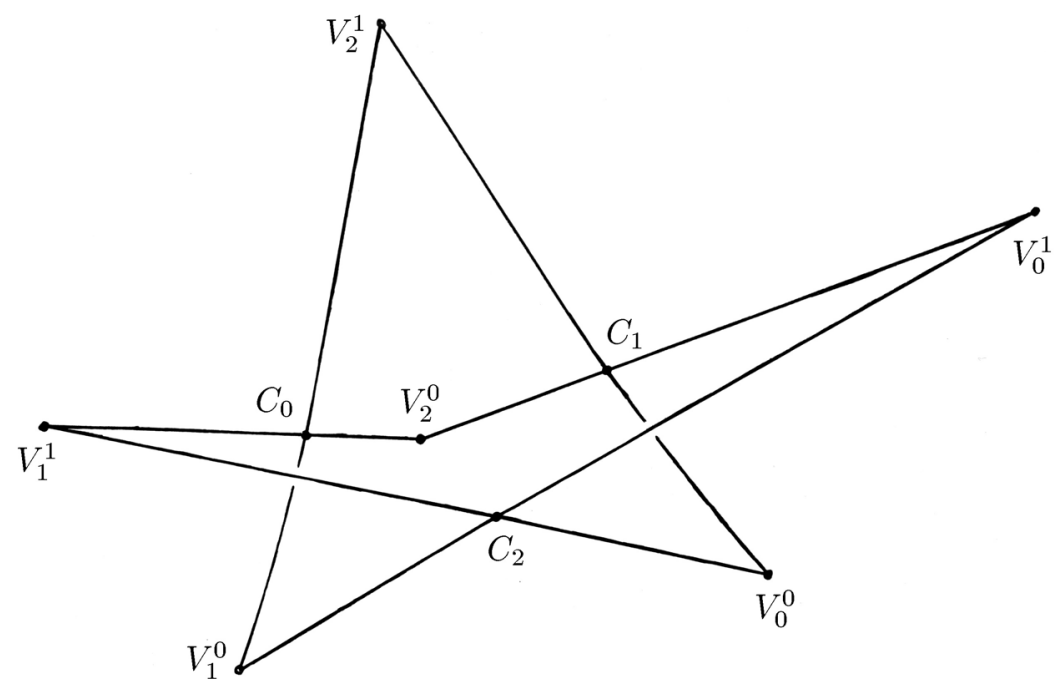

Рис. 3

ЗАмечание 6.6. Рис. 1 получен совмещением рис. 2 и рис. 3.

Рассмотрим ростки фронта $\Sigma$ в точках $C_{0}, C_{1}, C_{2}$. В силу лемм 6.2 и 6.5 они диффеоморфны ростку в 0 множества $\left\{y^{2}=x^{3}\right\} \cup\left\{z^{2}=x^{3}\right\}$ (росток фронта $\Sigma$ в точке $C_{2}$ изображен на рис. 4). При малой общей деформации кривой $Г$ из каждой точки $C_{0}, C_{1}, C_{2}$ возникают две точки возврата двойных линий фронта кривой. При этом двойная линия, входящая в точку возврата вдоль кривой $\eta$, выходит из нее вдоль одной из кривых $\xi_{1}, \xi_{2}, \xi_{3}$.

Отсюда видно, что росток фронта $\Sigma$ кривой $\Gamma$ в любой точке $C_{k}, k=0,1,2$, может быть продеформирован двумя разными способами. Следовательно, множество особых точек фронта любой кривой общего положения, достаточно близкой к $Г$, может быть получено из множества $S_{\Sigma}=\xi_{1} \cup \xi_{2} \cup \xi_{3} \cup \eta \cup \Gamma^{*}$ одной из 8 деформаций. Но множество $S_{\Sigma}$ инвариантно относительно отражения $(a, b, c) \rightarrow(a, b,-c)$ и вращений вокруг прямой $a=b=0$ на углы, кратные $2 \pi / 3$. Поэтому достаточно рассмотреть только две малые деформации фронта $\Sigma$ (они реализуются малыми деформациями кривой $Г$ ):

$\Theta_{1}$ : точки пересечения ветвей $V_{0}^{0} V_{1}^{1}, V_{2}^{0} V_{0}^{1}, V_{1}^{0} V_{2}^{1}\left(V_{0}^{1} V_{1}^{0}, V_{2}^{1} V_{0}^{0}, V_{1}^{1} V_{2}^{0}\right)$ кривой $\Gamma^{*}$ с плоскостью $c=0$ выходят из треугольника $C_{0} C_{1} C_{2}$ (входят внутрь треугольника соответственно); 
$\Theta_{2}$ : точки пересечения ветвей $V_{0}^{1} V_{1}^{0}, V_{2}^{0} V_{0}^{1}, V_{1}^{0} V_{2}^{1}\left(V_{0}^{0} V_{1}^{1}, V_{2}^{1} V_{0}^{0}, V_{1}^{1} V_{2}^{0}\right)$ кривой $\Gamma^{*}$ с плоскостью $c=0$ выходят из треугольника $C_{0} C_{1} C_{2}$ (входят внутрь треугольника соответственно).

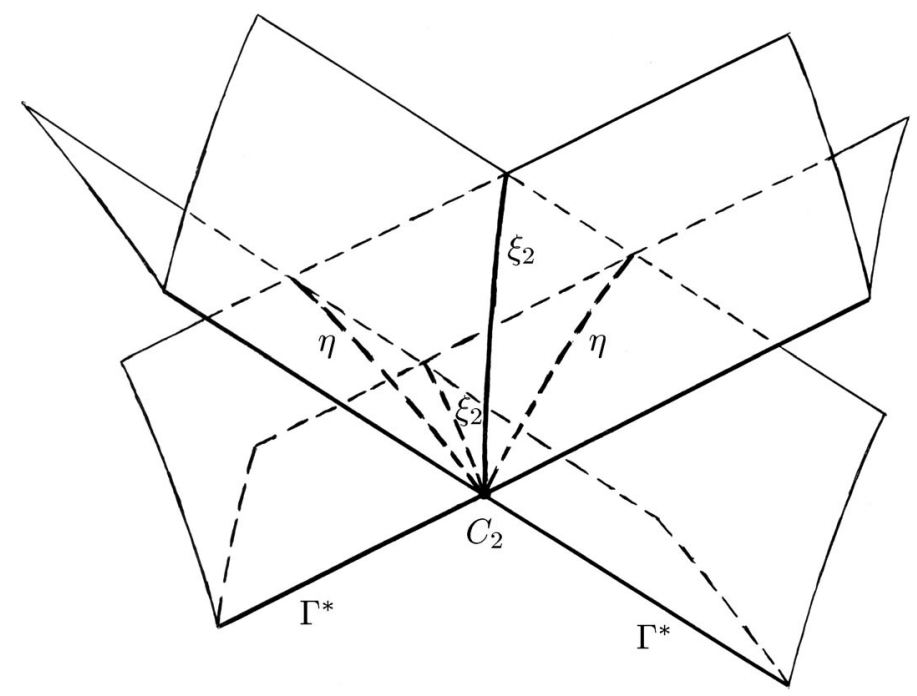

Рис. 4

Фронт, полученный из фронта $\Sigma$ одной из этих двух деформаций, имеет следующие двойные линии:

$$
\Theta_{1}:\left\{\begin{array}{l}
V_{0}^{0} \stackrel{\xi_{0}}{\longrightarrow} C_{0}^{-} \stackrel{\eta}{\longrightarrow} C_{1}^{-} \stackrel{\xi_{1}}{\longrightarrow} V_{1}^{1}, \\
V_{1}^{0} \stackrel{\xi_{1}}{\longrightarrow} \tilde{C}_{1}^{-} \stackrel{\eta}{\longrightarrow} C_{2}^{-} \stackrel{\xi_{2}}{\longrightarrow} V_{2}^{1}, \\
V_{2}^{0} \stackrel{\xi_{2}}{\longrightarrow} \tilde{C}_{2}^{-} \stackrel{\eta}{\longrightarrow} \tilde{C}_{0}^{-} \stackrel{\xi_{0}}{\longrightarrow} V_{0}^{1}
\end{array} ; \quad \Theta_{2}:\left\{\begin{array}{l}
V_{0}^{0} \stackrel{\xi_{0}}{\longrightarrow} C_{0}^{-} \stackrel{\eta}{\longrightarrow} C_{1}^{-} \stackrel{\xi_{1}}{\longrightarrow} V_{1}^{1}, \\
V_{1}^{0} \stackrel{\xi_{1}}{\longrightarrow} \tilde{C}_{1}^{-} \stackrel{\eta}{\longrightarrow} C_{2}^{+} \stackrel{\xi_{2}}{\longrightarrow} V_{2}^{0}, \\
V_{2}^{1} \stackrel{\xi_{2}}{\longrightarrow} \tilde{C}_{2}^{+} \stackrel{\eta}{\longrightarrow} \tilde{C}_{0}^{-} \stackrel{\xi_{0}}{\longrightarrow} V_{0}^{1} .
\end{array}\right.\right.
$$

Здесь мы обозначаем близкие вершины ласточкиных хвостов исходного и продеформированного фронтов одними и теми же буквами; стрелка означает, что двойная линия идет вдоль кривой, указанной над этой стрелкой; точки возврата двойных линий, появившиеся из данной точки $C_{k}$, обозначаются через $C_{k}^{ \pm}$ и $\widetilde{C}_{k}^{ \pm}$, причем знак $+(-)$означает, что данная точка возврата положительна (отрицательна) относительно ориентации двойной линии, указанной стрелками (ориентация пространства $\mathbb{R}^{3 *}$ задается координатами $\left.(a, b, c)\right)$.

Легко видеть, что

1) фронты, полученные из фронта $\Sigma$ деформациями $\Theta_{1}, \Theta_{2}$, не имеют замкнутых двойных линий; это доказывает предложение 2.8;

2) диаграммы главных уплощений кривых в $\mathbb{R}^{3}$ с фронтами, полученными из фронта $\Sigma$ деформациями $\Theta_{1}$ и $\Theta_{2}$, являются диаграммами, описанными в пп. 1) и 2) предложения 3.7 соответственно; таким образом, это предложение также доказано.

ЗАмЕЧАНИЕ 6.7. Допустимые гомотопии кривой сохраняют гомотопический тип двойственной кривой (в пространстве замкнутых кривых в $P^{3 *}$ без самопересе- 
чений, но с точками возврата). Если кривая общего положения $\gamma$, полученная допустимой гомотопией из кривой, достаточно близкой к $Г$, имеет непустую диаграмму главных уплощений, то ее двойственная кривая незаузлена. Если же кривая $\gamma$ имеет пустую диаграмму главных уплощений, то ее двойственная кривая изотопна трилистнику.

Автор благодарен В. А. Васильеву, В. В. Горюнову, М. Э. Казаряну и рецензенту за полезные обсуждения и замечания. Я хотел бы также выразить благодарность Ньютоновскому институту математики в Кембридже, где эта работа была частично написана, за гостеприимство и поддержку.

\section{ЛИТЕРАТУРА}

1. Арнольд В. И. К лежандровой теории Штурма пространственных кривых. Функц. анализ и его прил., 32, вып. 2, 1-7 (1998).

2. Арнольд В. И. Задачи Арнольда. Фазис, М., 2000.

3. Barner $M$. Über die Mindestanzahl stationärer Schmiegebenen bei geschlossenen strengkonvexen Raumkurven. Abh. Math. Sem. Univ. Hamburg, 20, 196-215 (1956).

4. Васильев B. A. Топология дополнений к дискриминантам. Фазис, М., 1997.

5. Седых В. Д. Строение выпуклой оболочки пространственной кривой. Труды семинара Петровского, 6, 239-256 (1981).

6. Седых B. Д. Теорема о четырех вершинах выпуклой пространственной кривой. Функц. анализ и его прил., 26, вып. 1, 35-41 (1992).

Российский государственный университет нефти и газа им. И. М. Губкина

Поступило в редакцию 20 марта 2000 г. 